\section{Liaison psychiatry and older adults}

Lloyd \& Mayou (2003) argue cogently for the development of liaison psychiatry, or 'psychological medicine in general hospitals' as they prefer it. Their arguments would carry more weight with commissioners if they included the mental health needs of older people. Two-thirds of general hospital beds are occupied by people aged over 65 and it is obvious to anyone visiting a general medical ward that the majority of 'bed-blocking' is caused by older people with dementia, depression and prolonged delirium. The epidemiology, together with demonstrably poor coordination of services, is highlighted in a recent report, partly commissioned by the Faculty of Old Age Psychiatry of the Royal College of Psychiatrists (Holmes et al, 2002).

The time is now ripe for old age psychiatrists to take their multi-disciplinary skills and experience of community support into general hospitals to enable earlier discharge of so-called bed blockers. Liaison psychiatrists could develop their services (and sub-speciality status) more quickly and comprehensively if they worked more closely with old age psychiatry colleagues clinically, and within the Royal College of Psychiatrists.

\section{Holmes, J., Bentley, K. \& Cameron, I. (2002) \\ Between Two Stools: Psychiatric Services for Older People in General Hospitals. Leeds: University of Leeds Academic} Unit of Psychiatry and Behavioural Sciences.

Lloyd, G. G. \& Mayou, R. A. (2003) Liaison psychiatry or psychological medicine? British Journal of Psychiatry, 183, 5-7.

J. Seymour Older Adults Mental Health Care Group, Michael Carlisle Centre, Nether Edge Hospital, 75 Osbourne Road, Sheffield SII 9BF, UK

\section{Liaison psychiatry and general hospital management}

In their excellent editorial Lloyd \& Mayou (2003) lament the lack of substantial progress in the development of liaison psychiatry. They refer to Scotland's greater cognisance of liaison psychiatry (Scottish Executive Health Department, 2000), before reaching practical conclusions, foremost among which is for liaison psychiatry services to be managed by the acute hospitals they serve. They also refer to Kendell's analysis of the physical-mental divide (Kendell, 2001). I wish to pick up these points.
As a mentor after I took up post in Edinburgh, Bob Kendell impressed on me his view that liaison psychiatrists were 'ambassadors of psychiatry' in the general hospital. He pointed out that, for most hospital specialists, liaison psychiatrists would be the only psychiatrists they were likely to encounter - so we had to be available, approachable, helpful, practical, considerate and sensible; we had to be good clinical psychiatrists and we had to resolve problems rather than cause them.

That does not mean liaison psychiatrists cannot bring special skills to the workplace, but it does imply that such skills have to be welded to basic clinical nous and rapport with colleagues. The significance of this is increased in a world in which we tend to be more valued by physicians than fellow psychiatrists - perhaps increasingly so as psychiatry becomes focused on severe and enduring mental illness, and other forms of disability, suffering and resource demand are marginalised.

Next, I made the leap from mental health to general hospital management when trusts were first mooted (without understanding the implications - it just felt right), and this turned out to be a fortuitous decision. Liaison psychiatry at the Infirmary rapidly expanded as I planned with medical colleagues solutions to the clinical problems we faced: some funds came through the Trust Improvement Programme and some from individual directorates seeking to purchase consultant sessions. I do not believe this initiative would, or could, have happened had I remained with the mental health unit. Now the rest of Scotland has progressed as the Executive's intentions have been made good - notably, with the appointment of four consultant liaison psychiatrists and 10.5 liaison nurses in Glasgow.

The consequences of going with the acute trust have been far-reaching. Psychiatry is not regarded as an alien speciality - we are seen as assets. We understand the environment and the pressures and we respond to the needs that arise rather than pursue a purist agenda. Crucially, we appreciate that what the acute hospital requires primarily from its psychiatric service lies in the areas of rapid assessment, immediate management and optimal resource usage rather than the proven areas of effectiveness that are highlighted as the rationale for spending on liaison psychiatry.

As a tangible illustration of our significance and role, the department of psychological medicine in Edinburgh's new Royal Infirmary has not been located up some back alley or on the top floor. We are sited on the ground floor near the hospital's front door because we are recognised as a key constituent in the modernisation agenda that has swept through acute medical care in the hospital. Without 'going native' I doubt that much of this progress would have occurred - so seek to make this management leap rather than sticking with tried and trusted strategies that have been found wanting.

Kendell, R. E. (200I) The distinction between mental and physical illness. British Journal of Psychiatry, I78, 490-493.

Lloyd, G. G. \& Mayou, R. A. (2003) Liaison psychiatry or psychological medicine? British Journal of Psychiatry, 183, 5-7.

Scottish Executive Health Department (2000) Our National Health. Edinburgh: Stationery Office.

G. Masterton Department of Psychological Medicine, Royal Infirmary of Edinburgh, 5I Little France Crescent, Edinburgh EHI6 4SA, UK

\section{Adverse events following neurosurgery}

Matthews \& Eljamel (2003) gave an excellent overview of the controversial field of neurosurgery for mental disorders (NMD). I agree with them that 'the accumulated literature on neurosurgery for mental disorder remains highly unsatisfactory' but would like to point to some recent evidence.

Matthews \& Eljamel state that 'there is surprisingly little evidence' to support the occurrence of adverse personality change. In my opinion, some of the literature suggests otherwise. Herner (1961) noted that in a group of 116 capsulotomy cases, frontal lobe deficit syndrome was obvious at follow-up in $30 \%$. In the anxiety group, $40 \%$ and $13 \%$, respectively, had adverse events of mild and of modest severity. In another study (Kullberg, 1977), capsulotomy caused 'some personality changes in the majority of the patients'. Adverse events in those studies included fatigue, emotional blunting, emotional incontinence, indifference, low initiative, disinhibition and impaired sense of judgement.

In a very recent study (Rück et al, 2003), 26 anxiety patients who had undergone bilateral thermocapsulotomy were followed up after a mean of 13 years. Psychiatric methods included symptom rating scales and neuropsychological testing. To 